INTERNATIONAL ENGINEERING, SCIENCE AND EDUCATION GROUP

\section{Middle East Journal of Science}

(2019) 5(1): 1-12

Published online June, 2019 (http://dergipark.gov.tr/mejs)

doi: 10.23884/mejs.2019.5.1.01

e-ISSN 2618-6136

Received: February 25, 2019 Accepted: April 11, 2019

Submission Type: Research Article

\title{
ON DECAY AND BLOW UP OF SOLUTIONS FOR A SYSTEM OF KIRCHHOFF TYPE EQUATIONS WITH DAMPING TERMS
}

\section{Erhan Pişkin*}

Dicle University, Department of Mathematics, Diyarbakır, Turkey; Orcid: 0000-0001-6587-4479

* Corresponding author: episkin@dicle.edu.tr

\begin{abstract}
In this paper, we investigate system of Kirchhoff type equations with bounded domain. We obtain decay of solutions by using multiplier method. Later, we will prove blow up results for negative inital energy.
\end{abstract}

Keywords: Decay, Blow up, Kirchhoff type equation.

Mathematics Subject Classification (2010): 35B40, 35B44.

\section{Introduction}

In this paper, we consider the following initial boundary value system

$$
\begin{cases}u_{t t}-M\left(\|\nabla u\|^{2}\right) \triangle u+\gamma_{2} u_{t}+\left|u_{t}\right|^{p} u_{t}=F_{u}(u, v), & (x, t) \in \Omega \times(0, T), \\ v_{t t}-M\left(\|\nabla v\|^{2}\right) \Delta v+\gamma_{2} v_{t}+\left|v_{t}\right|^{q} v_{t}=F_{v}(u, v), & (x, t) \in \Omega \times(0, T), \\ u(x, t)=v(x, t)=0, & (x, t) \in \partial \Omega \times(0, T), \\ u(x, 0)=u_{0}(x), u_{t}(x, 0)=u_{1}(x), & x \in \Omega, \\ v(x, 0)=v_{0}(x), v_{t}(x, 0)=v_{1}(x), & x \in \Omega,\end{cases}
$$

where $\Omega$ is a bounded domain with smooth boundary $\partial \Omega$ in $R^{n}(n=1,2,3), p, q>0, \gamma_{2}>0$. Let $\triangle=\sum_{j=1}^{n} \frac{\partial^{2}}{\partial x_{j}^{2}}$ be the Laplace operator, and $M(s)$ be a nonnegative locally Lipschitz function, and $F: R^{2} \longrightarrow R$ is a $C^{1}$ function given by

$$
F(u, v)=a|u+v|^{r+2}+2 b|u v|^{\frac{r+2}{2}},
$$

where $r \geq 2, a>1$ and $b>0$, which implies

$$
\begin{aligned}
& F_{u}(u, v)=(r+2)\left[a|u+v|^{r}(u+v)+b|u|^{\frac{r-2}{2}}|v|^{\frac{r+2}{2}} u\right], \\
& F_{v}(u, v)=(r+2)\left[a|u+v|^{r}(u+v)+b|v|^{\frac{r-2}{2}}|u|^{\frac{r+2}{2}} v\right] .
\end{aligned}
$$

Also, we have

$$
u F_{u}(u, v)+v F_{v}(u, v)=(r+2) F(u, v) \quad \forall(u, v) \in R^{2} .
$$


Lemma 1.1 [10]. Let $c_{0}$ and $c_{1}$ be positive constants, such that

$$
c_{0}\left(|u|^{r+2}+|v|^{r+2}\right) \leq F(u, v) \leq c_{1}\left(|u|^{r+2}+|v|^{r+2}\right) .
$$

Throughout this paper, we define $M(s)$ by

$$
M(s)=\alpha+\beta s^{\gamma}, s \geq 0, \alpha, \beta>0, \gamma \geq 0 .
$$

Obviously, $M(s)$ is a nonnegative locally Lipschitz function.

The problem (1.1) is a generalization of a model introduced by Kirchhoff [5]. More precisely, Kirchhoff proposed a model given by the equation for $f=g=0$,

$$
\rho h \frac{\partial^{2} u}{\partial t^{2}}+\delta \frac{\partial u}{\partial t}+g\left(\frac{\partial u}{\partial t}\right)=\left\{\rho_{0}+\frac{E h}{2 L} \int_{0}^{L}\left(\frac{\partial u}{\partial x}\right)^{2} d x\right\} \frac{\partial^{2} u}{\partial x^{2}}+f(u),
$$

for $0<x<L, t \geq 0$.

The qualitative analysis of solutions for a single Kirchhoff type equation

$$
u_{t t}-M\left(\|\nabla u\|^{2}\right) \triangle u+g\left(u_{t}\right)=f(u), x \in \Omega, t>0
$$

has been discussed by many authors, see [3, 7, 12, 18, 20].

When $M(s)=1,(1.1)$ become the following system

$$
\left\{\begin{array}{c}
u_{t t}-\triangle u+\gamma_{2} u_{t}+\left|u_{t}\right|^{p} u_{t}=F_{u}(u, v) \\
v_{t t}-\triangle v+\gamma_{2} v_{t}+\left|v_{t}\right|^{q} v_{t}=F_{v}(u, v)
\end{array}\right.
$$

Many authors studied the existence, blow up and decay of solutions of $(1.8)$ (see $[6,11,13$, 14, 19, 21]). Also, many authors studied the existence and nonexistence of solutions (1.8) with $\gamma_{2}=0([2,16,17])$.

Motivated by previous works, we study the decay of solutions and the blow up of solutions with negative initial energy for the system of Kirchhoff type equations with damping terms.

The outline of this paper is as follows. In section 2, we give some lemmas and notations. In section 3 , the decay of the solution is given. Section 4 , we show the blow up properties of solution.

\section{Preliminaries}

In this paper, $\|\cdot\|$ and $\|\cdot\|_{p}$ denote the usual $L^{2}(\Omega)$ norm and $L^{p}(\Omega)$ norm, respectively. Firstly, we give the following lemmas:

Lemma 2.1 [8]. Suppose that, $0<p(n=1,2)$ or $0<p \leq 2 \frac{n-1}{n-2}(n \geq 3)$ holds. Then there exists a positive constant $C>1$ depending only on $\Omega$, such that

$$
\|u\|_{p}^{s} \leq C\left(\|\nabla u\|^{2}+\|u\|_{p}^{p}\right)
$$

for any $u \in H_{0}^{1}(\Omega), 2 \leq s \leq p$. 
Lemma 2.2 (Sobolev-Poincare inequality) [1]. Let $p$ be a number with $2 \leq p<\infty(n=1,2)$ or $2 \leq p \leq 2 n /(n-2)(n \geq 3)$, such that

$$
\|u\|_{q} \leq C_{*}\|\nabla u\| \text { for } u \in H_{0}^{1}(\Omega)
$$

where $C_{*}=C_{*}(\Omega, q)$ is a constant.

We introduce the following functionals

$$
\begin{aligned}
J(t)= & J(u(t), v(t))=\frac{\alpha}{2}\left(\|\nabla u\|^{2}+\|\nabla v\|^{2}\right) \\
& +\frac{\beta}{2(\gamma+1)}\left(\|\nabla u\|^{2(\gamma+1)}+\|\nabla v\|^{2(\gamma+1)}\right)-\int_{\Omega} F(u, v) d x
\end{aligned}
$$

and

$$
\begin{aligned}
I(t)= & I(u(t), v(t))=\alpha\left(\|\nabla u\|^{2}+\|\nabla v\|^{2}\right) \\
& +\beta\left(\|\nabla u\|^{2(\gamma+1)}+\|\nabla v\|^{2(\gamma+1)}\right)-(r+2) \int_{\Omega} F(u, v) d x .
\end{aligned}
$$

Next, we introduce the energy functional

$$
\begin{aligned}
E(t)= & \frac{1}{2}\left(\left\|u_{t}\right\|^{2}+\left\|v_{t}\right\|^{2}\right)+\frac{\alpha}{2}\left(\|\nabla u\|^{2}+\|\nabla v\|^{2}\right) \\
& +\frac{\beta}{2(\gamma+1)}\left(\|\nabla u\|^{2(\gamma+1)}+\|\nabla v\|^{2(\gamma+1)}\right)-\int_{\Omega} F(u, v) d x .
\end{aligned}
$$

We also define

$$
W=\left\{(u, v):(u, v) \in\left(H_{0}^{1}(\Omega) \cap H^{2}(\Omega)\right) \times\left(H_{0}^{1}(\Omega) \cap H^{2}(\Omega)\right), I(u, v)>0\right\} \cup\{(0,0)\} .
$$

Lemma 2.3. Let $u(x, t)$ be the solution of (1.1). Then

$$
E^{\prime}(t)=-\gamma_{2}\left(\left\|u_{t}\right\|^{2}+\left\|v_{t}\right\|^{2}\right)-\left(\left\|u_{t}\right\|_{p+2}^{p+2}+\left\|v_{t}\right\|_{q+2}^{q+2}\right) \leq 0 .
$$

Proof. By multiplying the first equation of (1.1) by $u_{t}$ and the second equation by $v_{t}$, integrating over $\Omega$, we obtain

$$
E(t)-E(0)=-\int_{0}^{t}\left[\gamma_{2}\left(\left\|u_{\tau}\right\|^{2}+\left\|v_{\tau}\right\|^{2}\right)+\left(\left\|u_{\tau}\right\|_{p+2}^{p+2}+\left\|v_{\tau}\right\|_{q+2}^{q+2}\right)\right] d \tau \text { for } t \geq 0
$$

We state a local existence result without a proof here (see $[4,15,18])$

Theorem 2.1. Suppose that $\min \{p, q\}>r$ such that

$$
\left\{\begin{array}{c}
0<p, q, \quad 0<r, \quad n=1,2 \\
0<p, q \leq \frac{2}{n-2}, \quad 0<r, \quad n \geq 3,
\end{array}\right.
$$

and let $\left(u_{0}, v_{0}\right) \in\left(H_{0}^{1}(\Omega) \cap H^{2}(\Omega)\right) \times\left(H_{0}^{1}(\Omega) \cap H^{2}(\Omega)\right),\left(u_{1}, v_{1}\right) \in H_{0}^{1}(\Omega) \times H_{0}^{1}(\Omega)$ be given. Then the problem (1.1) has a unique local solution

$$
u, v \in C\left([0, T) ; H_{0}^{1}(\Omega) \cap H^{2}(\Omega)\right) \text { and } u_{t}, v_{t} \in C\left([0, T) ; H_{0}^{1}(\Omega)\right),
$$


for any fixed time $T>0$.

\section{The decay result}

In this section, we consider the energy decay of the solution to (1.1). For this purpose, we use the functional

$$
\Phi(t)=E(t)+\epsilon \int_{\Omega}\left(u u_{t}+v v_{t}\right) d x+\frac{\epsilon \gamma_{2}}{2}\left(\|u\|^{2}+\|v\|^{2}\right),
$$

where $\epsilon$ is a positive constant. Inspiring by idea in [9], we will show in the next lemma that $\Phi(t)$ and $E(t)$ are equivalent.

Lemma 3.1. For $\epsilon>0$ small enough, the relation

$$
\alpha_{1} E(t) \leq \Phi(t) \leq \alpha_{2}(E(t))^{\frac{1}{\gamma+1}}
$$

holds for two positive constants $\alpha_{1}$ and $\alpha_{2}$.

Proof. Applying Young inequality and Sobolev embedding theorem, we obtain

$$
\begin{aligned}
\Phi(t) & \leq E(t)+\frac{\epsilon}{2}\left(\int_{\Omega} u^{2} d x+\int_{\Omega} u_{t}^{2} d x+\int_{\Omega} v^{2} d x+\int_{\Omega} v_{t}^{2} d x\right)+\frac{\epsilon \gamma_{2}}{2}\left(\|u\|^{2}+\|v\|^{2}\right) \\
& \leq\left(1+\frac{\epsilon}{2}\right) E(t)+C_{*} \frac{\epsilon \gamma_{2}}{2} C^{\frac{1}{\gamma+1}}\left(\|\nabla u\|^{2(\gamma+1)}+\|\nabla v\|^{2(\gamma+1)}\right)^{\frac{1}{(\gamma+1)}}
\end{aligned}
$$

where $(a+b)^{\lambda} \leq C\left(a^{\lambda}+b^{\lambda}\right), a, b>0$ was used. After that we obtain

$$
\begin{aligned}
\Phi(t) & \leq\left(1+\frac{\epsilon}{2}\right) E(t)+C_{*} \frac{\epsilon \gamma_{2}}{2} C^{\frac{1}{\gamma+1}}(E(t))^{\frac{1}{(\gamma+1)}} \\
& \leq\left(\left(1+\frac{\epsilon}{2}\right)(E(t))^{\frac{\gamma}{\gamma+1}}+C_{*} \frac{\epsilon \gamma_{2}}{2} C^{\frac{1}{\gamma+1}}\right)(E(t))^{\frac{1}{(\gamma+1)}} \\
& \leq\left(\left(1+\frac{\epsilon}{2}\right)(E(0))^{\frac{\gamma}{\gamma+1}}+C_{*} \frac{\epsilon \gamma_{2}}{2} C^{\frac{1}{\gamma+1}}\right)(E(t))^{\frac{1}{(\gamma+1)}} \\
& =\alpha_{2}(E(t))^{\frac{1}{(\gamma+1)}}
\end{aligned}
$$

and

$$
\begin{aligned}
\Phi(t) & \geq E(t)-\epsilon\left[\frac{1}{4 \tau}\left(\left\|u_{t}\right\|^{2}+\left\|v_{t}\right\|^{2}\right)+\tau\left(\|u\|^{2}+\|v\|^{2}\right)\right]+\frac{\epsilon \gamma_{2}}{2}\left(\|u\|^{2}+\|v\|^{2}\right) \\
& \geq E(t)-\frac{\epsilon}{4 \tau}\left(\left\|u_{t}\right\|^{2}+\left\|v_{t}\right\|^{2}\right)+\epsilon\left(\frac{\gamma_{2}}{2}-\tau\right)\left(\|u\|^{2}+\|v\|^{2}\right) \\
& \geq E(t)-\frac{\epsilon}{4 \tau}\left(\left\|u_{t}\right\|^{2}+\left\|v_{t}\right\|^{2}\right) \\
& =J(t)+\left(\frac{1}{2}-\frac{\epsilon}{4 \tau}\right)\left(\left\|u_{t}\right\|^{2}+\left\|v_{t}\right\|^{2}\right) \\
& \geq J(t)+\frac{\alpha_{1}}{2}\left(\left\|u_{t}\right\|^{2}+\left\|v_{t}\right\|^{2}\right) \\
& \geq \alpha_{1} E(t) .
\end{aligned}
$$

for small enough $\tau$. This completes the proof.

Theorem 3.1. Suppose that $\min \{p, q, r\}>2 \gamma$ and

$$
\frac{\beta(2 \gamma+1)}{2(\gamma+1)}>2 c_{*}^{r+2} c_{1}(r+1)\left(\frac{2(r+2)(\gamma+1)}{\beta(r-2 \gamma)} E(0)\right)^{\frac{r-2 \gamma}{2(\gamma+1)}}
$$


such that $(2.5)$ is satisfied and let $\left(u_{0}, v_{0}\right) \in W$ be given. Then the solution satisfies

$$
E(t) \leq \begin{cases}K e^{-k t}, & \gamma=0, \\ (k t+K)^{-\frac{1}{\gamma}}, & \gamma>0,\end{cases}
$$

where $K$ and $k$ are positive constants which will be defined later.

Proof. Now differentiate (3.1) and use Eq. (1.1) and Young inequality, we have

$$
\begin{aligned}
\Phi^{\prime}(t)= & -\gamma_{2}\left(\left\|u_{t}\right\|^{2}+\left\|v_{t}\right\|^{2}\right)-\left(\left\|u_{t}\right\|_{p+2}^{p+2}+\left\|v_{t}\right\|_{q+2}^{q+2}\right)+\epsilon\left(\left\|u_{t}\right\|^{2}+\left\|v_{t}\right\|^{2}\right) \\
& -\epsilon \alpha\left(\|\nabla u\|^{2}+\|\nabla v\|^{2}\right)-\epsilon \beta\left(\|\nabla u\|^{2(\gamma+1)}+\|\nabla v\|^{2(\gamma+1)}\right) \\
& -\epsilon\left(\int_{\Omega} u u_{t}\left|u_{t}\right|^{p} d x+\int_{\Omega} v v_{t}\left|v_{t}\right|^{q} d x\right)+\epsilon(r+2) \int_{\Omega} F(u, v) d x \\
\leq & -\left(\gamma_{2}-\epsilon\right)\left(\left\|u_{t}\right\|^{2}+\left\|v_{t}\right\|^{2}\right)-\left(\left\|u_{t}\right\|_{p+2}^{p+2}+\left\|v_{t}\right\|_{q+2}^{q+2}\right) \\
& -\epsilon \alpha\left(\|\nabla u\|^{2}+\|\nabla v\|^{2}\right)-\epsilon \beta\left(\|\nabla u\|^{2(\gamma+1)}+\|\nabla v\|^{2(\gamma+1)}\right) \\
& +\epsilon\left(\delta\|u\|_{p+2}^{p+2}+c(\delta)\left\|u_{t}\right\|_{p+2}^{p+2}+\delta\|v\|_{q+2}^{q+2}+c(\delta)\left\|v_{t}\right\|_{q+2}^{q+2}\right) \\
& +\epsilon(r+2) \int_{\Omega} F(u, v) d x .
\end{aligned}
$$

By using the definition of the $E(t)$, we get

$$
\begin{aligned}
\Phi^{\prime}(t) \leq & -\epsilon E(t)-\left(\gamma_{2}-\frac{3 \epsilon}{2}\right)\left(\left\|u_{t}\right\|^{2}+\left\|v_{t}\right\|^{2}\right)-\frac{\epsilon \alpha}{2}\left(\|\nabla u\|^{2}+\|\nabla v\|^{2}\right) \\
& -(1-c(\delta))\left(\left\|u_{t}\right\|_{p+2}^{p+2}+\left\|v_{t}\right\|_{q+2}^{q+2}\right)+\epsilon\left(\delta\|u\|_{p+2}^{p+2}+\delta\|v\|_{q+2}^{q+2}\right) \\
& +\epsilon(r+1) \int_{\Omega} F(u, v) d x-\frac{\epsilon \beta(2 \gamma+1)}{2(\gamma+1)}\left(\|\nabla u\|^{2(\gamma+1)}+\|\nabla v\|^{2(\gamma+1)}\right) .
\end{aligned}
$$

By use of $F(u, v) \leq c_{1}\left(|u|^{r+2}+|v|^{r+2}\right)$, we have

$$
\begin{gathered}
\Phi^{\prime}(t) \leq-\epsilon E(t)+\epsilon \delta\left(\|u\|_{p+2}^{p+2}+\|v\|_{q+2}^{q+2}\right)+\epsilon c_{1}(r+1)\left(\|u\|_{r+2}^{r+2}+\|v\|_{r+2}^{r+2}\right) \\
-\frac{\epsilon \beta(2 \gamma+1)}{2(\gamma+1)}\left(\|\nabla u\|^{2(\gamma+1)}+\|\nabla v\|^{2(\gamma+1)}\right) .
\end{gathered}
$$

Since $I(t)>0$,

$$
\begin{aligned}
J(t) & =\frac{r}{2(r+2)}\left[\alpha\left(\|\nabla u\|^{2}+\|\nabla v\|^{2}\right)+\frac{\beta(r-2 \gamma)}{(\gamma+1) r}\left(\|\nabla u\|^{2(\gamma+1)}+\|\nabla v\|^{2(\gamma+1)}\right)\right]+\frac{1}{r+2} I(t) \\
& \geq \frac{r}{2(r+2)}\left[\alpha\left(\|\nabla u\|^{2}+\|\nabla v\|^{2}\right)+\frac{\beta(r-2 \gamma)}{(\gamma+1) r}\left(\|\nabla u\|^{2(\gamma+1)}+\|\nabla v\|^{2(\gamma+1)}\right)\right] .
\end{aligned}
$$

Thus,

$$
\begin{aligned}
\|\nabla u\|^{2(\gamma+1)}+\|\nabla v\|^{2(\gamma+1)} & \leq \frac{2(r+2)(\gamma+1)}{\beta(r-2 \gamma)} J(t) \\
& \leq \frac{2(r+2)(\gamma+1)}{\beta(r-2 \gamma)} E(t) \\
& \leq \frac{2(r+2)(\gamma+1)}{\beta(r-2 \gamma)} E(0) .
\end{aligned}
$$


From Poincare inequality, we have

$$
\begin{aligned}
\|u\|_{p+2}^{p+2} & \leq c_{*}^{p+2}\|\nabla u\|^{p+2} \\
& =c_{*}^{p+2}\|\nabla u\|^{p-2 \gamma}\|\nabla u\|^{2(\gamma+1)} \\
& =c_{*}^{p+2}\left(\|\nabla u\|^{2(\gamma+1)}\right)^{\frac{p-2 \gamma}{2(\gamma+1)}}\|\nabla u\|^{2(\gamma+1)} \\
& \leq c_{*}^{p+2}\left(\frac{2(r+2)(\gamma+1)}{\beta(r-2 \gamma)} E(0)\right)^{\frac{p-2 \gamma}{2(\gamma+1)}}\|\nabla u\|^{2(\gamma+1)},
\end{aligned}
$$

and similarly

$$
\|v\|_{q+2}^{q+2} \leq c_{*}^{q+2}\left(\frac{2(r+2)(\gamma+1)}{\beta(r-2 \gamma)} E(0)\right)^{\frac{q-2 \gamma}{2(\gamma+1)}}\|\nabla v\|^{2(\gamma+1)}
$$

Furthermore,

$$
\|u\|_{r+2}^{r+2} \leq c_{*}^{r+2}\left(\frac{2(r+2)(\gamma+1)}{\beta(r-2 \gamma)} E(0)\right)^{\frac{r-2 \gamma}{2(\gamma+1)}}\|\nabla u\|^{2(\gamma+1)}
$$

and similarly

$$
\|v\|_{r+2}^{r+2} \leq c_{*}^{r+2}\left(\frac{2(r+2)(\gamma+1)}{\beta(r-2 \gamma)} E(0)\right)^{\frac{r-2 \gamma}{2(\gamma+1)}}\|\nabla v\|^{2(\gamma+1)} .
$$

Substituting (3.9)-(3.13) into (3.7), we have

$$
\begin{aligned}
\Phi^{\prime}(t) \leq & -\epsilon E(t)-\epsilon\left[\frac{\beta(2 \gamma+1)}{2(\gamma+1)}-2 c_{*}^{r+2} c_{1}(r+1)\left(\frac{2(r+2)(\gamma+1)}{\beta(r-2 \gamma)} E(0)\right)^{\frac{r-2 \gamma}{2(\gamma+1)}}\right. \\
& -\delta m]\left(\|\nabla u\|^{2(\gamma+1)}+\|\nabla v\|^{2(\gamma+1)}\right)
\end{aligned}
$$

where $m=\max \left\{c_{*}^{p+2}\left(\frac{2(r+2)(\gamma+1)}{\beta(r-2 \gamma)} E(0)\right)^{\frac{p-2 \gamma}{2(\gamma+1)}}, c_{*}^{q+2}\left(\frac{2(r+2)(\gamma+1)}{\beta(r-2 \gamma)} E(0)\right)^{\frac{q-2 \gamma}{2(\gamma+1)}}\right\}$. Thus from the assumptions of the theorem and by choosing a sufficiently small $\delta>0$, we obtain that

$$
\Phi^{\prime}(t) \leq-\epsilon E(t) \leq-\frac{\epsilon}{\left(\alpha_{2}\right)^{\gamma+1}}(\Phi(t))^{\gamma+1} .
$$

We separate (3.15) into two cases.

Case 1: $\gamma=0$, then a simply integration of $(3.15)$ over $(0, t)$ yields

$$
E(t) \leq \Phi(t) \leq \Phi(0) e^{-k t}
$$

where $k=\frac{\epsilon}{\left(\alpha_{2}\right)^{\gamma+1}}$.

Case 2: $\gamma>0$, a simply integration of $(3.15)$ over $(0, t)$ yields

$$
E(t) \leq \Phi(t) \leq\left(k t+\Phi^{-\gamma}(0)\right)^{-\frac{1}{\gamma}},
$$

where $k=\frac{\epsilon \gamma}{\left(\alpha_{2}\right)^{\gamma+1}}$. This completes the proof.

\section{Blow up}

In this section, we state and prove blow up result. 
Theorem 4.1. Suppose that $r>\max \{2 \gamma, p, q\}, E(0)<0$, and there exists a constant $\tau$ such that $\tau \leq \frac{2 \alpha \gamma}{\gamma_{2} C_{*}}$, where $C_{*}$ is the constant of the Sobolev embedding theorem. Then the solution of this system blows up in finite time $T^{*}$, and

$$
T^{*} \leq \frac{1-\sigma}{\xi \sigma \Psi^{\frac{\sigma}{1-\sigma}}(0)}
$$

where $\Psi(t)$ and $\sigma$ are given in (4.1) and (4.2) respectively.

Proof. Define $H(t)=-E(t)$, then $E(0)<0$ and $(2.6)$ gives $H(t) \geq H(0)>0$. Define

$$
\Psi(t)=H^{1-\sigma}(t)+\varepsilon\left(\int_{\Omega} u u_{t} d x+\int_{\Omega} v v_{t} d x\right)
$$

where $\varepsilon$ is a small constant to be chosen later and

$$
0<\sigma \leq \min \left\{\frac{r-p}{(r+2)(p+1)}, \frac{r-q}{(r+2)(q+1)}\right\} .
$$

A direct differentiation of $\Psi(t)$ gives

$$
\begin{aligned}
\Psi^{\prime}(t)= & (1-\sigma) H^{-\sigma}(t) H^{\prime}(t)+\varepsilon\left(\left\|u_{t}\right\|^{2}+\left\|v_{t}\right\|^{2}\right)-\varepsilon \alpha\left(\|\nabla u\|^{2}+\|\nabla v\|^{2}\right) \\
& -\varepsilon \beta\left(\|\nabla u\|^{2(\gamma+1)}+\|\nabla v\|^{2(\gamma+1)}\right)+\varepsilon(r+2) \int_{\Omega} F(u, v) d x \\
& -\varepsilon \gamma_{2}\left(\int_{\Omega} u u_{t} d x+\int_{\Omega} v v_{t} d x\right)-\varepsilon\left(\int_{\Omega} u u_{t}\left|u_{t}\right|^{p} d x+\int_{\Omega} v v_{t}\left|v_{t}\right|^{q} d x\right) .
\end{aligned}
$$

From definition of $H(t)$, it follows that

$$
\begin{aligned}
-\beta\left(\|\nabla u\|^{2(\gamma+1)}+\|\nabla v\|^{2(\gamma+1)}\right)= & 2(\gamma+1) H(t)+(\gamma+1)\left(\left\|u_{t}\right\|^{2}+\left\|v_{t}\right\|^{2}\right) \\
& +\alpha(\gamma+1)\left(\|\nabla u\|^{2}+\|\nabla v\|^{2}\right) \\
& -2(\gamma+1) \int_{\Omega} F(u, v) d x .
\end{aligned}
$$

Substitute (4.4) into (4.3) to obtain

$$
\begin{aligned}
\Psi^{\prime}(t)= & (1-\sigma) H^{-\sigma}(t) H^{\prime}(t)+\varepsilon\left(\left\|u_{t}\right\|^{2}+\left\|v_{t}\right\|^{2}\right) \\
& -\varepsilon \alpha\left(\|\nabla u\|^{2}+\|\nabla v\|^{2}\right)+2 \varepsilon(\gamma+1) H(t) \\
& +\varepsilon(\gamma+1)\left(\left\|u_{t}\right\|^{2}+\left\|v_{t}\right\|^{2}\right)+\varepsilon \alpha(\gamma+1)\left(\|\nabla u\|^{2}+\|\nabla v\|^{2}\right) \\
& -2 \varepsilon(\gamma+1) \int_{\Omega} F(u, v) d x+\varepsilon(r+2) \int_{\Omega} F(u, v) d x \\
& -\varepsilon \gamma_{2}\left(\int_{\Omega} u u_{t} d x+\int_{\Omega} v v_{t} d x\right)-\varepsilon\left(\int_{\Omega} u u_{t}\left|u_{t}\right|^{p} d x+\int_{\Omega} v v_{t}\left|v_{t}\right|^{q} d x\right) .
\end{aligned}
$$

Now, we make use of the following Young's inequality

$$
X Y \leq \frac{\delta^{k} X^{k}}{k}+\frac{\delta^{l} Y^{l}}{l},
$$

$X, Y \geq 0, \delta>0, k, l \in R^{+}$such that $\frac{1}{k}+\frac{1}{l}=1$. We have

$$
\int_{\Omega} u u_{t} d x \leq \frac{\tau}{2}\|u\|^{2}+\frac{1}{2 \tau}\left\|u_{t}\right\|^{2}, \quad \int_{\Omega} v v_{t} d x \leq \frac{\tau}{2}\|v\|^{2}+\frac{1}{2 \tau}\left\|v_{t}\right\|^{2},
$$




$$
\begin{aligned}
\int_{\Omega} u u_{t}\left|u_{t}\right|^{p} d x & \leq \frac{\delta_{1}^{p+2}}{p+2}\|u\|_{p+2}^{p+2}+\frac{(p+1) \delta_{1}^{-\frac{p+2}{p+1}}}{p+2}\left\|u_{t}\right\|_{p+2}^{p+2} \\
& \leq \frac{\delta_{1}^{p+2}}{p+2}\|u\|_{p+2}^{p+2}+\frac{(p+1) \delta_{1}^{-\frac{p+2}{p+1}}}{p+2} H^{\prime}(t)
\end{aligned}
$$

and similarly

$$
\int_{\Omega} v v_{t}\left|v_{t}\right|^{q} d x \leq \frac{\delta_{2}^{q+2}}{q+2}\|v\|_{q+2}^{q+2}+\frac{(q+1) \delta_{2}^{-\frac{q+2}{q+1}}}{q+2} H^{\prime}(t),
$$

where $\delta_{1}, \delta_{2}$ are constants depending on the time $t$ that will be specified later. Thus, (4.5) becomes

$$
\begin{aligned}
\Psi^{\prime}(t) \geq & (1-\sigma) H^{-\sigma}(t) H^{\prime}(t)+\varepsilon\left(\left\|u_{t}\right\|^{2}+\left\|v_{t}\right\|^{2}\right) \\
& +\varepsilon \alpha \gamma\left(\|\nabla u\|^{2}+\|\nabla v\|^{2}\right)+2 \varepsilon(\gamma+1) H(t)+\varepsilon(\gamma+1)\left(\left\|u_{t}\right\|^{2}+\left\|v_{t}\right\|^{2}\right) \\
& +\varepsilon(r-2 \gamma) \int_{\Omega} F(u, v) d x-\frac{\varepsilon \gamma_{2} \tau}{2}\left(\|u\|^{2}+\|v\|^{2}\right)-\frac{\varepsilon \gamma_{2}}{2 \tau}\left(\left\|u_{t}\right\|^{2}+\left\|v_{t}\right\|^{2}\right) \\
& -\varepsilon\left(\frac{(p+1) \delta_{1}^{-\frac{p+2}{p+1}}}{p+2}+\frac{(q+1) \delta_{2}^{-\frac{q+2}{q+1}}}{q+2}\right) H^{\prime}(t)-\varepsilon\left(\frac{\delta_{1}^{p+2}}{p+2}\|u\|_{p+2}^{p+2}+\frac{\delta_{2}^{q+2}}{q+2}\|v\|_{q+2}^{q+2}(k)^{\prime} 6\right)
\end{aligned}
$$

By using Sobolev-Poincare's inequality, we have

$$
\begin{aligned}
\Psi^{\prime}(t) \geq & (1-\sigma) H^{-\sigma}(t) H^{\prime}(t)+\varepsilon\left(\alpha \gamma-\frac{\gamma_{2} \tau C_{*}}{2}\right)\left(\|\nabla u\|^{2}+\|\nabla v\|^{2}\right)+2 \varepsilon(\gamma+1) H(t) \\
& +\varepsilon\left(\gamma+2-\frac{\gamma_{2}}{2 \tau}\right)\left(\left\|u_{t}\right\|^{2}+\left\|v_{t}\right\|^{2}\right)+\varepsilon(r-2 \gamma) \int_{\Omega} F(u, v) d x \\
& -\varepsilon\left(\frac{(p+1) \delta_{1}^{-\frac{p+2}{p+1}}}{p+2}+\frac{(q+1) \delta_{2}^{-\frac{q+2}{q+1}}}{q+2}\right) H^{\prime}(t)-\varepsilon\left(\frac{\delta_{1}^{p+2}}{p+2}\|u\|_{p+2}^{p+2}+\frac{\delta_{2}^{q+2}}{q+2}\|v\|_{q+2}^{q+2}(\text { f.. }\right. \\
&
\end{aligned}
$$

Therefore by taking $\delta_{1}$ and $\delta_{2}$ so that $\delta_{1}^{-\frac{p+2}{p+1}}=k_{1} H^{-\sigma}(t), \delta_{2}^{-\frac{q+2}{q+1}}=k_{2} H^{-\sigma}(t)$, where $k_{1}, k_{2}>0$ are specified later, we get

$$
\delta_{1}^{p+2}=k_{1}^{-(p+1)} H^{\sigma(p+1)}(t) \leq k_{1}^{-(p+1)} c_{1}^{\sigma(p+1)}\left(\|u\|_{r+2}^{r+2}+\|v\|_{r+2}^{r+2}\right)^{\sigma(p+1)},
$$

and

$$
\delta_{2}^{q+2}=k_{2}^{-(q+1)} H^{\sigma(q+1)}(t) \leq k_{2}^{-(q+1)} c_{1}^{\sigma(q+1)}\left(\|u\|_{r+2}^{r+2}+\|v\|_{r+2}^{r+2}\right)^{\sigma(q+1)},
$$

since $H(t)=-E(t) \leq \int_{\Omega} F(u, v) d x \leq c_{1}\left(|u|^{r+2}+|u|^{r+2}\right)$. 
Substituting (4.8) and (4.9) into (4.7), we have

$$
\begin{aligned}
\Psi^{\prime}(t) \geq & \left(1-\sigma-\frac{\varepsilon(p+1) k_{1}}{p+2}-\frac{\varepsilon(q+1) k_{2}}{q+2}\right) H^{-\sigma}(t) H^{\prime}(t)+2 \varepsilon(\gamma+1) H(t) \\
& +\varepsilon\left(\alpha \gamma-\frac{\gamma_{2} \tau C_{*}}{2}\right)\left(\|\nabla u\|^{2}+\|\nabla v\|^{2}\right) \\
& +\varepsilon\left(2+\gamma-\frac{\gamma_{2}}{2 \tau}\right)\left(\left\|u_{t}\right\|^{2}+\left\|v_{t}\right\|^{2}\right)+\varepsilon(r-2 \gamma) \int_{\Omega} F(u, v) d x \\
& -\frac{\varepsilon k_{1}^{-(p+1)} c_{1}^{\sigma(p+1)}}{p+2}\left(\|u\|_{r+2}^{r+2}+\|v\|_{r+2}^{r+2}\right)^{\sigma(p+1)}\|u\|_{p+2}^{p+2} \\
& -\frac{\varepsilon k_{2}^{-(q+1)} c_{1}^{\sigma(q+1)}}{q+2}\left(\|u\|_{r+2}^{r+2}+\|v\|_{r+2}^{r+2}\right)^{\sigma(q+1)}\|v\|_{q+2}^{q+2} .
\end{aligned}
$$

Since $r>\max \{p, q\}$, we obtain

$$
\begin{gathered}
\|u\|_{p+2}^{p+2} \leq C\|u\|_{r+2}^{p+2} \leq C\left(\|u\|_{r+2}+\|v\|_{r+2}\right)^{p+2}, \\
\|v\|_{q+2}^{q+2} \leq C\|v\|_{r+2}^{q+2} \leq C\left(\|u\|_{r+2}+\|v\|_{r+2}\right)^{q+2} .
\end{gathered}
$$

Thus

$$
\begin{aligned}
\Psi^{\prime}(t) \geq & \left(1-\sigma-\frac{\varepsilon(p+1) k_{1}}{p+2}-\frac{\varepsilon(q+1) k_{2}}{q+2}\right) H^{-\sigma}(t) H^{\prime}(t)+2 \varepsilon(\gamma+1) H(t) \\
& +\varepsilon\left(\alpha \gamma-\frac{\gamma_{2} \tau C_{*}}{2}\right)\left(\|\nabla u\|^{2}+\|\nabla v\|^{2}\right) \\
& +\varepsilon\left(2+\gamma-\frac{\gamma_{2}}{2 \tau}\right)\left(\left\|u_{t}\right\|^{2}+\left\|v_{t}\right\|^{2}\right)+\varepsilon(r-2 \gamma) \int_{\Omega} F(u, v) d x \\
& -\frac{\varepsilon k_{1}^{-(p+1)} c_{1}^{\sigma(p+1)} C}{p+2}\left(\|u\|_{r+2}+\|v\|_{r+2}\right)^{\sigma(r+2)(p+1)+p+2} \\
& -\frac{\varepsilon k_{2}^{-(q+1)} c_{1}^{\sigma(q+1)} C}{q+2}\left(\|u\|_{r+2}+\|v\|_{r+2}\right)^{\sigma(r+2)(q+1)+q+2},
\end{aligned}
$$

where $(a+b)^{\lambda} \leq C\left(a^{\lambda}+b^{\lambda}\right), a, b>0$ is used. From (4.2), we have $2 \leq \sigma(p+1)(r+2)+p+2 \leq$ $r+2,2 \leq \sigma(q+1)(r+2)+q+2 \leq r+2$. By using Lemma 2.1, we have

$$
\begin{gathered}
\|u\|_{r+2}^{\sigma(p+1)(r+2)+p+2} \leq C\left(\|\nabla u\|^{2}+\|u\|_{r+2}^{r+2}\right), \\
\|v\|_{r+2}^{\sigma(q+1)(r+2)+q+2} \leq C\left(\|\nabla v\|^{2}+\|v\|_{r+2}^{r+2}\right) .
\end{gathered}
$$

Thus

$$
\begin{aligned}
\Psi^{\prime}(t) \geq & \left(1-\sigma-\frac{\varepsilon(p+1) k_{1}}{p+2}-\frac{\varepsilon(q+1) k_{2}}{q+2}\right) H^{-\sigma}(t) H^{\prime}(t)+2 \varepsilon(\gamma+1) H(t) \\
& +\varepsilon\left(\alpha \gamma-\frac{\gamma_{2} \tau C_{*}}{2}\right)\left(\|\nabla u\|^{2}+\|\nabla v\|^{2}\right) \\
& +\varepsilon\left(2+\gamma-\frac{\gamma_{2}}{2 \tau}\right)\left(\left\|u_{t}\right\|^{2}+\left\|v_{t}\right\|^{2}\right)+\varepsilon(r-2 \gamma) \int_{\Omega} F(u, v) d x \\
& +\varepsilon\left(-\frac{k_{1}^{-(p+1)} c_{1}^{\sigma(p+1)} C}{p+2}-\frac{k_{2}^{-(q+1)} c_{1}^{\sigma(q+1)} C}{q+2}\right)\left(\|u\|_{r+2}^{r+2}+\|v\|_{r+2}^{r+2}\right) \\
& +\varepsilon\left(-\frac{k_{1}^{-(p+1)} c_{1}^{\sigma(p+1)} C}{p+2}-\frac{k_{2}^{-(q+1)} c_{1}^{\sigma(q+1)} C}{q+2}\right)\left(\|\nabla u\|^{2}+\|\nabla v\|^{2}\right) .
\end{aligned}
$$


By using the $c_{0}\left(|u|^{r+2}+|v|^{r+2}\right) \leq F(u, v)$ in (4.12) we obtain

$$
\begin{aligned}
\Psi^{\prime}(t) \geq & \left(1-\sigma-\frac{\varepsilon(p+1) k_{1}}{p+2}-\frac{\varepsilon(q+1) k_{2}}{q+2}\right) H^{-\sigma}(t) H^{\prime}(t) \\
& +2 \varepsilon(\gamma+1) H(t)+\varepsilon\left(2+\gamma-\frac{\gamma_{2}}{2 \tau}\right)\left(\left\|u_{t}\right\|^{2}+\left\|v_{t}\right\|^{2}\right) \\
& +\varepsilon\left(c_{0}(r-2 \gamma)-\frac{k_{1}^{-(p+1)} c_{1}^{\sigma(p+1)} C}{p+2}-\frac{k_{2}^{-(q+1)} c_{1}^{\sigma(q+1)} C}{q+2}\right)\left(\|u\|_{r+2}^{r+2}+\|v\|_{r+2}^{r+2}\right) \\
& +\varepsilon\left(\alpha \gamma-\frac{\gamma_{2} \tau C_{*}}{2}-\frac{k_{1}^{-(p+1)} c_{1}^{\sigma(p+1)} C}{p+2}-\frac{k_{2}^{-(q+1)} c_{1}^{\sigma(q+1)} C}{q+2}\right)\left(\|\nabla u\|^{2}+\|\nabla v\|^{2}(4,13)\right.
\end{aligned}
$$

where $r>2 \gamma$ is used. We choose $k_{1}, k_{2}$ large enough so that

$$
c_{0}(r-2 \gamma)-\frac{k_{1}^{-(p+1)} c_{1}^{\sigma(p+1)} C}{p+2}-\frac{k_{2}^{-(q+1)} c_{1}^{\sigma(q+1)} C}{q+2}>\frac{c_{0}(r-2 \gamma)}{2}
$$

and

$$
\alpha \gamma-\frac{\gamma_{2} \tau C_{*}}{2}-\frac{k_{1}^{-(p+1)} c_{1}^{\sigma(p+1)} C}{p+2}-\frac{k_{2}^{-(q+1)} c_{1}^{\sigma(q+1)} C}{q+2}>\frac{\alpha \gamma}{2}-\frac{\gamma_{2} \tau C_{*}}{4} .
$$

Then, we choose $\varepsilon$ small enough so that $1-\sigma-\frac{\varepsilon(p+1) k_{1}}{p+2}-\frac{\varepsilon(q+1) k_{2}}{q+2} \geq 0$. Thus, we have

$$
\begin{aligned}
\Psi^{\prime}(t) \geq & \varepsilon\left(2+\gamma-\frac{\gamma_{2}}{2 \tau}\right)\left(\left\|u_{t}\right\|^{2}+\left\|v_{t}\right\|^{2}\right)+2 \varepsilon(\gamma+1) H(t) \\
& +\varepsilon\left(\frac{\alpha \gamma}{2}-\frac{\gamma_{2} \tau C_{*}}{4}\right)\left(\|\nabla u\|^{2}+\|\nabla v\|^{2}\right)+\varepsilon \frac{c_{0}(r-2 \gamma)}{2}\left(\|u\|_{r+2}^{r+2}+\|v\|_{r+2}^{r+2}\right) \\
\geq & \eta\left(\left\|u_{t}\right\|^{2}+\left\|v_{t}\right\|^{2}+H(t)+\|\nabla u\|^{2}+\|\nabla v\|^{2}+\|u\|_{r+2}^{r+2}+\|v\|_{r+2}^{r+2}\right),
\end{aligned}
$$

where $\eta=\min \left\{\varepsilon\left(2+\gamma-\frac{\gamma_{2}}{2 \tau}\right), 2 \varepsilon(\gamma+1), \varepsilon\left(\frac{\alpha \gamma}{2}-\frac{\gamma_{2} \tau C_{*}}{4}\right), \varepsilon \frac{c_{0}(r-2 \gamma)}{2}\right\}$. Consequently we have

$$
\Psi(t) \geq \Psi(0)=H^{1-\sigma}(0)+\varepsilon\left(\int_{\Omega} u_{0} u_{1} d x+\int_{\Omega} v_{0} v_{1} d x\right)>0, \forall t \geq 0 .
$$

On the other hand, by the Hölder's inequality, we get

$$
\begin{aligned}
\left|\int_{\Omega} u u_{t} d x+\int_{\Omega} v v_{t} d x\right|^{\frac{1}{1-\sigma}} & \leq\|u\|^{\frac{1}{1-\sigma}}\left\|u_{t}\right\|^{\frac{1}{1-\sigma}}+\|v\|^{\frac{1}{1-\sigma}}\left\|v_{t}\right\|^{\frac{1}{1-\sigma}} \\
& \leq C\left(\|u\|_{r+2}^{\frac{1}{1-\sigma}}\left\|u_{t}\right\|^{\frac{1}{1-\sigma}}+\|v\|_{r+2}^{\frac{1}{1-\sigma}}\left\|v_{t}\right\|^{\frac{1}{1-\sigma}}\right) .
\end{aligned}
$$

Young inequality gives

$$
\left|\int_{\Omega} u u_{t} d x+\int_{\Omega} v v_{t} d x\right|^{\frac{1}{1-\sigma}} \leq C\left(\|u\|_{r+2}^{\frac{\mu}{1-\sigma}}+\left\|u_{t}\right\|^{\frac{\theta}{1-\sigma}}+\|v\|_{r+2}^{\frac{\mu}{1-\sigma}}+\left\|v_{t}\right\|^{\frac{\theta}{1-\sigma}}\right),
$$

for $\frac{1}{\mu}+\frac{1}{\theta}=1$. We take $\theta=2(1-\sigma)$, to get $\mu=\frac{2(1-\sigma)}{1-2 \sigma} \leq r+2$ by (4.2). Therefore (4.17) becomes

$$
\left|\int_{\Omega} u u_{t} d x+\int_{\Omega} v v_{t} d x\right|^{\frac{1}{1-\sigma}} \leq C\left(\left\|u_{t}\right\|^{2}+\left\|v_{t}\right\|^{2}+\|u\|_{r+2}^{\frac{2}{1-2 \sigma}}+\|v\|_{r+2}^{\frac{2}{1-2 \sigma}}\right) .
$$


By using Lemma 2.1, we obtain

$$
\left|\int_{\Omega} u u_{t} d x+\int_{\Omega} v v_{t} d x\right|^{\frac{1}{1-\sigma}} \leq C\left(\left\|u_{t}\right\|^{2}+\left\|v_{t}\right\|^{2}+\|u\|_{r+2}^{r+2}+\|v\|_{r+2}^{r+2}+\|\nabla u\|^{2}+\|\nabla v\|^{2}\right) .
$$

Thus

$$
\begin{aligned}
\Psi^{\frac{1}{1-\sigma}}(t) & =\left[H^{1-\sigma}(t)+\varepsilon\left(\int_{\Omega} u u_{t} d x+\int_{\Omega} v v_{t} d x\right)\right]^{\frac{1}{1-\sigma}} \\
& \leq 2^{\frac{\sigma}{1-\sigma}}\left(H(t)+\varepsilon^{\frac{1}{1-\sigma}}\left|\int_{\Omega} u u_{t} d x+\int_{\Omega} v v_{t} d x\right|^{\frac{1}{1-\sigma}}\right) \\
& \leq C\left(\left\|u_{t}\right\|^{2}+\left\|v_{t}\right\|^{2}+H(t)+\|u\|_{r+2}^{r+2}+\|v\|_{r+2}^{r+2}+\|\nabla u\|^{2}+\|\nabla v\|^{2}\right) .
\end{aligned}
$$

By combining of (4.14) and (4.20) we arrive

$$
\Psi^{\prime}(t) \geq \xi \Psi^{\frac{1}{1-\sigma}}(t)
$$

where $\xi$ is a positive constant. A simple integration yields

$$
\Psi^{\frac{\sigma}{1-\sigma}}(t) \geq \frac{1}{\Psi^{-\frac{\sigma}{1-\sigma}}(0)-\frac{\xi \sigma t}{1-\sigma}},
$$

which implies that the solution blows up in a finite time $T^{*}$, with

$$
T^{*} \leq \frac{1-\sigma}{\xi \sigma \Psi^{\frac{\sigma}{1-\sigma}}(0)}
$$

\section{References}

[1] Adams, R.A., Fournier, J.J.F., Sobolev Spaces, Academic Press, New York, 2003.

[2] Agre, K., Rammaha, M.A., "Systems of nonlinear wave equations with damping and source terms", Diff. Integral Eqns., 19, 1235-1270, 2006.

[3] Benaissa, A., Messaoudi, S.A., "Blow-up of solutions for the Kirchhoff equation of qLaplacian type with nonlinear dissipation", Colloquium Mathematicum, 94, 103-109, 2002.

[4] Georgiev, V., Todorova, G., "Existence of a solution of the wave equation with nonlinear damping and source term", J. Differ. Equations, 109 (1994) 295-308.

[5] Kirchhoff, G., Mechanik, Teubner, Leipzig, 1883.

[6] Korpusov, M.O., "Blow up the solution of a nonlinear system of equations with positive energy", Theoretical and Mathematical Physics, 171, 725-738, 2012.

[7] Matsuyama, T., Ikehata, R., "On global solutions and energy decay for the wave equations of the Kirchhoff type with nonlinear damping terms", J. Math. Anal. Appl., 204, 729-753, 1996. 
[8] Messaoudi, S.A., "Blow up in a nonlinearly damped wave equation", Math. Nachr., 231, 105-111, 2001.

[9] Messaoudi, S.A., "On the decay of solutions for a class of quasilinear hyperbolic equations with non-linear damping and source terms", Mat. Meth. Appl. Sci., 28, 1819-1828, 2005.

[10] Messaoudi, S.A., Said-Houari, B., "Global nonexistence of positive initial-energy solutions of a system of nonlinear viscoelastic wave equations with damping and source terms", $J$. Math. Anal. Appl., 365, 277-287, 2010.

[11] Miranda, M.M., Medeiros, L.A., "On the existence of global solutions of a coupled nonlinear Klein-Gordon equations", Funkcial Ekvac., 30, 147-161, 1987.

[12] Ono, K., "Global existence, decay, and blow-up of solutions for some mildly degenerate nonlinear Kirchhoff strings", J. Differential Equations, 137, 273-301, 1997.

[13] Pişkin, E., "Uniform decay and blow-up of solutions for coupled nonlinear Klein-Gordon equations with nonlinear damping terms", Math. Meth. Appl. Sci., 37, 3036-3047, 2014.

[14] Pişkin, E., "Blow-up of solutions for coupled nonlinear Klein-Gordon equations with weak damping terms", Math. Sci. Letters, 3, 189-191, 2014.

[15] Pişkin, E., "Existence, decay and blow up of solutions for the extensible beam equation with nonlinear damping and source terms", Open Math., 13, 408-420, 2015.

[16] Said-Houari, B., "Global nonexistence of positive initial-energy solutions of a system of nonlinear wave equations with damping and source terms", Diff. Integral Eqns., 23, 79-92, 2010.

[17] Said-Houari, B., "Global existence and decay of solutions of a nonlinear system of wave equations", Appl. Anal., 91, 475-489, 2012.

[18] Taniguchi, T., "Existence and asymptotic behaviour of solutions to weakly damped wave equations of Kirchhoff type with nonlinear damping and source terms", J. Math. Anal. Appl., 361, 566-578, 2010.

[19] Wu, S.T., "Blow-up results for system of nonlinear Klein-Gordon equations with arbitrary positive initial energ", Electron. J. Diff. Equations, 2012, 1-13, 2012.

[20] Wu, S.T., Tsai, L.Y., "Blow-up solutions for some nonlinear wave equations of Kirchhoff type with some dissipation", Nonlinear Anal., 65, 243-264, 2006.

[21] Ye, Y., "Global existence and asymptotic stability for coupled nonlinear Klein-Gordon equations with nonlinear damping terms", Dynamical Syst., 28, 287-298, 2013. 